\title{
EI Uso de las TIC en la Educación Superior: Aplicación a los Docentes de la Unidad de Admisión y Nivelación de la Escuela Superior Politécnica de Chimborazo
}

\author{
Germán Torres, \\ Magister en Interconectividad de Redes \\ Escuela Superior Politécnica de Chimborazo, Ecuador \\ Marco Salazar, \\ Magister en Gestión Empresarial \\ Escuela Superior Politécnica de Chimborazo, Ecuador \\ Gabriel Pilaguano, \\ Magister en Pequeñas y Medianas Empresas Mención Finanzas \\ Escuela Superior Politécnica de Chimborazo, Ecuador \\ Gabriela Fonseca, \\ Magister en Gestión de Proyectos de Desarrollo \\ Escuela Superior Politécnica de Chimborazo, Ecuador
}

Doi:10.19044/esj.2019.v15n19p72～URL:http://dx.doi.org/10.19044/esj.2019.v15n19p72

\section{Resumen}

El presente trabajo permite establecer el uso que los docentes de la Unidad de Admisión y Nivelación de la Escuela Superior Politécnica de Chimborazo, le dan a la plataforma virtual en sus actividades académicas permitiendo tener una idea clara de qué tipo de información circula a través de dicha plataforma. Este estudio genera herramientas que permiten que los estudiantes y docentes de la Unidad de Admisión y Nivelación de la Escuela Superior Politécnica de Chimborazo UAN tengan una comunicación efectiva. La metodología utilizada es la sistemática que recolecta información de un grupo seleccionado de personas, la misma que nos permite recopilar datos primarios de los encuestados. Se obtiene como resultado de la aplicación de la técnica de campo y la encuesta que el 63,4\% de los docentes utilizan sus aulas virtuales como herramienta de apoyo en sus actividades académicos, que el 61 $\%$ de los docentes realiza un seguimiento al acceso de los recursos que publican y los cuales son utilizados por sus estudiantes en las aulas virtuales en la ESPOCH. La universalización de la utilización de las TICs es evidente, ya que en el Ecuador, según datos del Ministerio de Telecomunicaciones, un $91 \%$ de la población entre 14 y50 años hace uso diariamente de dichas 
herramientas de comunicación a través de sus dispositivos inteligentes. Por tal motivo, con este estudio se pretende concientizar a los docentes de la Unidad de Admisión y Nivelación de la ESPOCH sobre el uso de la Plataforma, 97,6\% de los docentes indican que el uso de las TIC puede mejorar el proceso de enseñanza con el uso del aula virtual. También se establece lineamientos para mejorar la comunicación fuera del aula de clase hacia los estudiantes con fines académicos, transmitiendo por medio de las aulas virtuales información de calidad en donde el docente se convierta en el tutor guía de las asignaturas que se imparten en la Unidad de Admisión de la ESPOCH, garantizando así métodos de respuesta inmediata desde el docente hacia el estudiante y vice versa.

Palabras clave: TIC, Educación Superior, ESPOCH, Unidad de Admisión y Nivelación, Aula virtual

\title{
The Use of ICT in Higher Education: Application to the Teachers of the Admission and Leveling Unit of the Chimborazo Politécnica Superior School
}

\author{
Germán Torres, \\ Magister en Interconectividad de Redes \\ Escuela Superior Politécnica de Chimborazo, Ecuador \\ Marco Salazar, \\ Magister en Gestión Empresarial \\ Escuela Superior Politécnica de Chimborazo, Ecuador \\ Gabriel Pilaguano, \\ Magister en Pequeñas y Medianas Empresas Mención Finanzas \\ Escuela Superior Politécnica de Chimborazo, Ecuador \\ Gabriela Fonseca, \\ Magister en Gestión de Proyectos de Desarrollo \\ Escuela Superior Politécnica de Chimborazo, Ecuador
}

\begin{abstract}
The paper focuses on the use of ICT in higher education using a case study of teachers of the Admission and Leveling Unit at the Polytechnic School of Chimborazo. A virtual platform was used in the course of their academic activities which allow them to have a clear idea of what type of
\end{abstract}


information circulates through the said platform. This study generates tools that allow students and teachers of the Admission and Leveling Unit of the Higher Polytechnic School of Chimborazo to have an effective communication. The methodology used in this study is a system that collects information from a selected group of people. It also allows them to collect primary data from the respondents. The application of the field technique and the survey technique shows that $63.4 \%$ of teachers use their virtual classrooms as a support tool in their academic activities. $61 \%$ of teachers monitor access to the resources they publish and are used by their students in virtual classrooms at ESPOCH. The universalization of the use of ICT is evident since in Ecuador, according to data from the Ministry of Telecommunications, $91 \%$ of the population between 14 - 50 years make use of these communication tools daily through their smart devices. Therefore, this study is aimed at raising the awareness of the teachers of the Admission and Leveling Unit of the ESPOCH on the use of the Platform. $97.6 \%$ of teachers indicate that the use of ICT can improve the teaching process through the use of the virtual classroom. Guidelines are also established to improve communication outside the classroom to students with academic purposes, transmitting quality information through the virtual classrooms where the teacher becomes the tutor of the subjects taught in the Unit. Admission of the ESPOCH, thus, guarantees immediate response methods from the teacher to the student and vice versa.

Keywords: ICT, Higher Education, ESPOCH, Admission and Leveling Unit, Virtual Classroom

\section{Introducción}

Las Tecnologías de la Información y Comunicación (TIC) están provocando profundos cambios en las esferas económica, política y social en función de sus diversas modalidades de presentación en el mundo, cambios que han incentivado su exploración conceptual en distintos ámbitos de competencia (Lira, 2005). De acuerdo con Bazzo (2010) las TIC brindan condiciones óptimas para transformar una enseñanza tradicional, pasiva, y fundamentalmente centrada en la transmisión del contenido, el profesor y la clase, en otro tipo de educación más personalizada, participativa, centrada en alcanzar aprendizajes diversos y que posea una real significación para cada estudiante.

En los últimos años, las Tecnologías de la Información y la Comunicación (TIC) se han convertido en un elemento esencial en los nuevos espacios de interacción del ser humano (Coll, 2004; Monereo \& Pozo, 2007). En este sentido, las TIC han logrado insertarse en la sociedad actual y transformarla de acuerdo a sus funcionalidades y alcances, teniendo incluso 
un impacto significativo en el ámbito educativo (Benvenuto, 2003; Carneiro, Toscano \& Díaz, 2009; Coll, 2004; Jaramillo, Castañeda \& Pimienta, 2009; Pedró, 2011).

El hacer frente a la integración de las TIC como soporte para la innovación requiere hacer frente a la reorganización de prácticas educativas que ya están consolidadas y, por lo tanto, enfrentar los sentimientos de ambigüedad, incertidumbre y riesgo que ello conlleva; es por esta razón que se habla de un proceso dinámico de reflexión continua, en el que las tecnologías dentro del campo educativo deben ser analizadas en un primer momento como instrumentos para la enseñanza y el aprendizaje (BanannanRitland, 2008). De acuerdo con Tilve, Gewerc, y Álvarez (2009) la introducción de herramientas tecnológicas para mejorar los procesos de enseñanza y aprendizaje es insuficiente si ésta no se produce en el contexto de una formulación metodológica global (formativa, organizativa y curricular), capaz de generar algún tipo de ruptura con los modelos educativos previos.

Lo anteriormente expuesto va en concordancia con De Pablos Pons \& Jiménez Cortés (2007), quienes manifiestan que en la actualidad resulta fácil constatar que, independientemente de los niveles educativos que queramos identificar, la formación y actualización del profesorado tiene lugar ineludiblemente al incorporar el uso de las tecnologías de la información y la comunicación, por tanto, en el ámbito de la educación superior, los responsables de las instituciones universitarias hace tiempo que han incorporado planes de dotación de infraestructuras tecnológicas e iniciativas formativas que fomenten el uso de dichas tecnologías por parte de los docentes.

Dentro de las dimensiones de la innovación en TIC desde la política educativa debe existir un proceso de cambio que incida en las formas de construcción del conocimiento, en la configuración de nuevos entornos de enseñanza-aprendizaje y en la transformación de la cultura escolar y docente. La innovación educativa en TIC es concebida como un proceso de cambio amplio que impacta a tres niveles: social, institucional y curricular, todo ello con la finalidad de la mejora educativa. Este planteamiento está relacionado con el concepto de "calidad educativa" y de él se derivan competencias profesionales para la formación inicial de maestros (De Pablos Pons \& Jiménez Cortés, 2007).

Y es que, en definitiva, como afirma Area (2000), lo relevante será el desarrollo de procesos formativos dirigidos a que cualquier sujeto aprenda a aprender (es decir, adquiera las habilidades para el autoaprendizaje de modo permanente a lo largo de su vida); sepa enfrentarse a la información (buscar, seleccionar, elaborar y difundir aquella información que sea necesaria y útil); se cualifique laboralmente para el uso de las nuevas tecnologías de la información y comunicación y tome conciencia de las implicaciones 
económicas, ideológicas, políticas y culturales de la tecnología en nuestra sociedad.

Bajo este contexto el presente artículo tiene como objetivo analizar el uso de las tecnologías de la información y comunicación por parte de los docentes de la Unidad de Admisión y Nivelación de la Escuela Superior Politécnica de Chimborazo.

\section{Metodología}

El enfoque metodológico empleado es el sistemático que recolecta información de un grupo seleccionado de personas, el mismo que nos permite recopilar datos primarios de los encuestados. Los datos que se obtienen son confiables, porque las respuestas se limitan a lo planteado. La interpretación de los mismos es sencilla y se utiliza este tipo de método ya que su estudio se aplica a muestras pequeñas.

Para la confección del cuestionario se realizó una búsqueda de la literatura sobre el tema que nos ocupa. El instrumento final quedó compuesto por 10 ítems, distribuidos en: Si los docentes utilizan las aulas virtuales (3 items), Uso y preferencia de contenido de las aulas (3 items), Aplicación y seguimiento de contenidos de la plataforma virtual para fines académicos (4 items).

La aplicación del banco de preguntas se realizó el pasado lunes 15 de enero a los docentes de las diferentes áreas que conforman las carreras de la UAN pertenecientes al marco muestral, lo que nos ha permitido conocer la percepción del uso de las aulas virtuales que los docentes en la Unidad de Admisión y Nivelación cuyos resultados se detallan a continuación:.

\section{Desarrollo}

Las Tecnologías de la Información y la Comunicación (TIC)

Hilbert y Katz (2003), definen a las Tecnologías de la Información y Comunicación como un sistema tecnológico conformado por cuatro componentes básicos:

a) infraestructura física,

b) software,

c) estructuras de comunicaciones y mecanismos de coordinación, y

d) contenidos.

$\mathrm{Su}$ uso permite que las personas puedan comunicarse y compilar, generar, manejar, transferir y divulgar información desde puntos geográficos distantes, por medio de desarrollo de hardware y software especializados. El resultado de la utilización de las TIC conlleva a la digitalización de datos, información y conocimientos en formatos digitales de texto, imágenes, sonidos, voz u otros, los cuales son almacenados, manejados, procesados y difundidos en el denominado proceso informacional. 


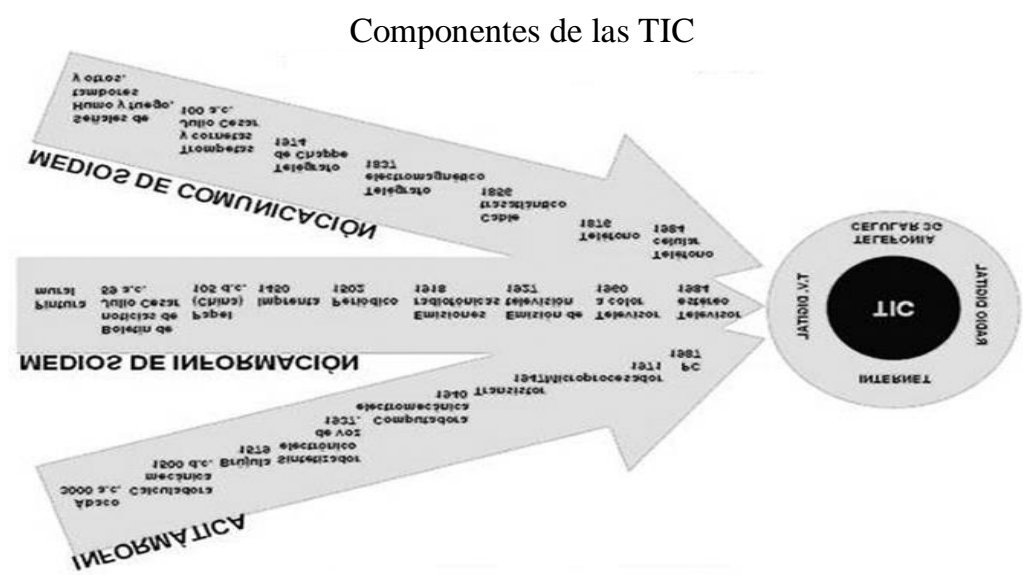

Fuente: (Hilbert \& Katz, 2003)

Por su parte (Linares, 2004), manifiesta que las Tecnologías de la Información y Comunicación, como parte del fenómeno tecnológico contemporáneo, pueden ser exploradas por medio de cuatro modalidades:

a) herramienta u objeto técnico;

b) mecanismo de aprendizaje y de generación de conocimientos;

c) un conjunto de actividades especializadas; y

d) medio para ejercer una voluntad colectiva de dominación y control.

Del mismo modo Las Tecnologías de la Información y Comunicación (TIC), pueden ser definidas como el conjunto de "tecnologías para el almacenamiento, recuperación, proceso y comunicación de la información" (Belloch, 2012).

Como objeto técnico, en la construcción de la sociedad de la información, la CEPAL(2003) identifica a las TIC como la convergencia entre las trayectorias tecnológicas de los medios de información, de comunicación y de informática: Internet, telefonía celular 3G, televisión digital y asistentes digitales personales.

Así también, según la UNESCO (2013), al referirse a las Tecnologías de la Información y la Comunicación (TIC), estas han tenido un desarrollo explosivo en la última parte del siglo XX y el comienzo del siglo XXI, al punto de que han dado forma a lo que se denomina «Sociedad del Conocimiento»o «de la Información». Prácticamente no hay un solo ámbito de la vida humana que no se haya visto impactado por este desarrollo: la salud, las finanzas, los mercados laborales, las comunicaciones, el gobierno, la productividad industrial, etc. El conocimiento se multiplica más rápido que nunca antes y se distribuye de manera prácticamente instantánea.

Al realizar un análisis de las tecnologías de la información y comunicación en el Ecuador Gonzales Nelo, Trelles César, y Mora Janeth (2017) manifiestan que se han desarrollado políticas para facilitar la 
incorporación de las TIC en el área educativa. Para Peñaherrera (2012) las TIC se han integrado mediante dotación de infraestructura, software educativo, equipamiento de aulas con recursos informáticos, capacitación al profesorado, creación de portales educativos, soporte técnico a las escuelas, entre otros. Durante el 2002 se ejecutó el programa maestr@s.com, dirigido a docentes en ejercicio, el cual consistió en la entrega de computadores personales y capacitación en el manejo de suite ofimática. En el 2007, se implementó el plan de las unidades del milenio, específicamente en las zonas rurales, como parte integral de la política gubernamental para mejorar la calidad de la educación pública, incorporando edificaciones con recursos pedagógicos adecuados, equipamiento moderno y tecnología avanzada (Ministerio de Educación, 2007). En el 2014, el Ministerio de Educación entregó kits tecnológicos a docentes de instituciones educativas. De acuerdo a la planificación la meta del proyecto fue integrar a la comunidad educativa en línea a un total de 5.500 instituciones educativas y entregar computadoras portátiles con servicio de conectividad a 147.170 docentes de todo el país (Ministerio de Educación , 2014). El currículo de los niveles de educación obligatoria por su parte fomenta el uso de las TIC en todas las áreas de estudio; por ejemplo, en el área de Matemática plantea utilizar los nuevos recursos tecnológicos para favorecer el quehacer matemático (Ministerio de Eduación, 2016). De igual forma prevé diseñar estrategias para el uso de las TIC en el abordaje de las diferentes áreas de estudio. En los últimos años el Ministerio de Educación mediante su programa de Formación Continua ha venido ofertando cursos de capacitación en las diferentes disciplinas a los docentes del magisterio fiscal, cursos que han sido planificados para que sean dictados en modalidad semipresencial y virtual con una duración de 330 horas, de las cuales 60 estaban destinadas específicamente a capacitación en el manejo de TIC.

Es difícil hablar de procesos de enseñanza - aprendizaje sin pensar en el uso de las TIC, pero no sólo como un complemento, sino como la vía de cambio cultural que permita mejorar la calidad de la educación, ahorrando tiempo en procesos mecánicos y profundizando aspectos relevantes como conceptualizaciones e interpretaciones argumentadas. Al respecto Kumari y D'Souza (2016) señalan que las TIC usadas apropiadamente, desarrollan la creatividad y habilidades de pensamiento de orden superior en los estudiantes, habilidades que son esenciales en el siglo XXI.

Por tanto, la incorporación de las TIC exige una redefinición de los roles de docentes y estudiantes. Los docentes deben convertirse en asesores, colaboradores y dinamizadores del aprendizaje mediante una acción tutorial direccionada a seleccionar los programas de formación y diagnosticar sus necesidades y problemas académicos. Los estudiantes por su parte deben formar parte activa del proceso de enseñanza a través de la búsqueda, 
intercambio e interpretación de la información, fomentando su autonomía (Salinas, 2008).

Es importante también indicar que no basta con la dotación de infraestructura tecnológica, sino que también es necesario ejecutar políticas que propongan dar un tratamiento integral al uso de las TIC en educación pues "la tecnología informática por sí misma no genera aprendizaje de forma espontánea, sino que depende de los fines educativos, de los métodos didácticos y de las actividades que realizan los alumnos con los ordenadores en el aula" (Area, 2007). En este sentido Wengslinky (1998) a través de la prueba National Assessment of Educational Progress (NAEP) en Estados Unidos, concluyó que cuando los profesores estaban lo suficientemente capacitados para dirigir a los estudiantes, el uso de la tecnología parecía estar asociado con mejoras significativas en los logros de aprendizaje en matemáticas.

\section{Las TIC en el Ámbito Educativo}

Hoy en día existe una sociedad en constante cambio, a la cual algunos autores como Castells (2001) denominan Sociedad de la Información o Sociedad del Conocimiento; es decir, una sociedad que se caracteriza por el cúmulo de información a su alcance y cuyo interés es poder administrar en forma eficiente dicha información, lo que se traduce en poder analizar, sintetizar y tomar decisiones acertadas considerando el manejo dela misma. Es por ello que las Tecnologías de la Información y la Comunicación (TIC) se han convertido en elementos cotidianos e imprescindibles de nuestra sociedad: tecnologías que determinan "no sólo la forma de comunicación sino también la economía, las instituciones políticas, la cultura y en definitiva la forma de aprender y valorar el mundo" (Cabero, 2004).

La utilización de las nuevas tecnologías de la información y la comunicación, y en concreto el uso de Internet, en los diferentes niveles educativos es una realidad palpable que queda de manifiesto en los numerosos proyectos y experiencias educativas innovadoras que cada año se dan a conocer a través de las jornadas, congresos y revistas. Los avances que se dan en la educación superior universitaria no son una excepción y prueba de ello es el aumento de artículos y publicaciones dirigidas a esta etapa en los que se suele percibir la siguiente base común: en la medida en que se introducen nuevos recursos tecnológicos se abre la puerta al cambio de aspectos pedagógicos (Area, Problemas y retos educativos ante las tecnologías digitales en la sociedad de la información, 2000).

La sociedad actual exige al docente de cualquier nivel educativo una competencia referida al uso de las Tecnologías de la Información y Comunicación (TIC). La importancia de esta competencia deriva del hecho de que es una competencia básica para que el profesor pueda desenvolverse con 
éxito en las diferentes funciones de su práctica educativa (Pedraza, Lavín, \& Torres, 2013). El profesor universitario no es la excepción, ya que las TIC se están incorporando en los distintos procesos educativos que estos dirigen. El profesor como agente de cambio y colaborador en la transformación social debería estar capacitado para reducir la brecha digital y el analfabetismo tecnológico (Gómez \& Cano, 2011). Esto sitúa a los docentes universitarios ante desafíos profesionales que implican desarrollar habilidades y capacidades en el uso e incorporación de estas nuevas tecnologías, sobre todo porque la calidad de la enseñanza y el aprendizaje están relacionadas con la formación del profesorado.

La aparición de las Tecnologías de la Información y Comunicación (TIC) irrumpe de lleno en la educación de las personas, no solamente como un medio de acceso a los recursos docentes, sino también en los procesos de aprendizaje; es decir, tanto en la forma de enseñar como en la forma de aprender (Cascales, 2017).

Para Domínguez(2011) la formación en el uso de las TIC es la clave para el perfeccionamiento e incorporación de estos medios tecnológicos en la enseñanza para así reducir con esto la brecha digital en el sistema educativo. Dicha formación comienza con la formación inicial y se extiende a través de la formación continua. Sin embargo López de la y Chávez (2013) mencionan que, si bien la formación docente en TIC debe ser un proceso permanente de adquisición y desarrollo de conocimientos y habilidades, también debe ser de nuevas actitudes ante una educación más flexible y abierta, indispensable para que los estudiantes de hoy se interesen por analizar y comprender su realidad y puedan satisfacer sus necesidades formativas de manera acorde con su estructura cognitiva. En medio de esto, hay que considerar sus propios límites, perspectivas y visión de cambio.

Sin embargo, en la actualidad existe una brecha importante en torno a las destrezas y habilidades para el manejo de recursos tecnológicos presentes en profesores y estudiantes. Es lo que se denomina «brecha digital», que algunos autores como Serrano (2003) definen como: la separación que existe entre las personas (comunidades, estados, países) que utilizan las Tecnologías de Información y Comunicación como una parte rutinaria de su vida diaria y aquellas que no tienen acceso a las mismas y que aunque las tengan no saben cómo utilizarlas.

Las necesidades de formación de los docentes en el uso de las TIC se centran en dos tipos de formación: tecnológica y pedagógica. En la primera las precisiones principales de los docentes son capacitación en el uso de software libre, en el manejo de la computadora, de procesadores de textos, hojas de cálculo y navegación en Internet. En el segundo tipo de formación la mayoría de los docentes requieren formación en la elaboración de materiales didácticos, uso de las TIC para que los estudiantes las empleen en el desarrollo 
de actividades de aprendizaje y manejo didáctico de plataformas educativas virtuales (Jiménez et al., 2015). Esto confirma la necesidad del profesorado de aplicar pedagógicamente estas tecnologías en los procesos de enseñanza y aprendizaje (Márquez \& Ramón, 2013) al igual que de integrarse a los nuevos ambientes de aprendizaje que genera la educación a distancia.

La mayoría de los docentes poseen las competencias tecnológicas, sin embargo, es una necesidad para ellos mantenerse actualizados constantemente, de tal manera que puedan estar a la vanguardia tecnológica, mejorar su desempeño docente, facilitar el proceso de enseñanza y aprendizaje, además de cumplir con las exigencias políticas educativas (Jiménez et al., 2015).

\section{Las TIC como herramienta en la Educación Superior}

De acuerdo con Benvenuto (2003), la tecnología seguirá aportando nuevas herramientas para el uso docente. La presión tecnológica no cesará, y la respuesta a ella no es necesariamente "llenar" los centros de educación superior de computadores personales para corregir la inequidad de acceso a Internet, sino que también deben desarrollarse en los académicos las habilidades y destrezas en el uso de TIC. Es un imperativo de primera magnitud conocer a través de la capacitación gradual, formal y sistemática, cuáles son las ventajas, usos y alcances de la tecnología antes de emprender su uso motivado por la modernidad o el aparente atractivo.

Las TIC están adquiriendo el carácter de imprescindible en los tiempos actuales por la alta competencia en la enseñanza superior, las demandas de calidad, y la globalización en el acceso. La incorporación de las TIC en el ambiente universitario y en la docencia, en particular, debe ser el resultado de una adecuada planificación que considere el rediseño del proceso docente, con nuevos roles, estructuras, currículos y metodologías. En general, se observa un cierto distanciamiento de parte importante de los académicos en el uso sistemático de TIC; esta situación puede también verse explicada por la ausencia de métodos de evaluación de las mismas en la docencia superior.

El uso adecuado de las TIC supone en primer lugar talleres o seminarios de actualización, posteriormente actividades complementarias para incorporarlas y mantenerlas en el proceso. Dadas las condiciones vigentes y lo observado en los últimos años, se estima que pocos académicos estarán dispuestos a realizar este esfuerzo adicional. En estas actividades tecnodocentes, por llamarlas de alguna forma, se invierte un considerable tiempo y no es cierto que sean evaluadas y consideradas adecuadamente en los procedimientos de evaluación correspondiente. Una razón muy simple, entre otras, es que los mecanismos de evaluación suponen la actividad docente sólo en el aula tradicional. 
Incluso para el rol docente clásico, no se observa que se considere en general para efectos de evaluaciones y promociones si el académico logra con calidad los objetivos docentes. Los mecanismos de evaluación y promoción privilegian aspectos de investigación y de dirección, no menos importantes, por sobre aspectos docentes. La ausencia de métricas o mecanismos que midan y premien la calidad del proceso docente implica que el académico privilegie aquellas actividades de mayor "rentabilidad" en términos de evaluación y promoción. Dicho criterio por cierto debe ser revisado en pro de mejorar la calidad del proceso docente en la enseñanza superior. Tal observación debe atenderse con especial premura en atención a la disposición, conocimiento, uso y cercanía a las tecnologías de información que tienen las nuevas y creativas generaciones.

La Unesco ha implementado las normas sobre competencias en TIC para docentes, pero si se observan bien los conceptos que maneja, también se pueden aplicar a los estudiantes o a cualquier persona que se encuentre en un entorno educativo. Los docentes tienen que ser competentes al concebir comunidades del conocimiento basadas en las TIC, y también deben saber utilizar estas tecnologías para fomentar las competencias de los estudiantes en materia de creación de conocimientos, así como su aprendizaje permanente y reflexivo.

Es importante considerar que todas las prácticas de enseñanza y aprendizaje deben evaluarse de diferentes maneras; por ejemplo, las nuevas herramientas e instrumentos necesarios para supervisar los logros y progresos realizados en el contexto de las TIC. En este aspecto no hay una posición clara $\mathrm{y}$ definida en los indicadores, instrumentos y escalas de medición (Scheuermann, Kikis \& Villalba, 2009).

Las principales competencias que se deben considerar se presentan a continuación:

\begin{tabular}{|l|l|l|}
\hline Competencias & Habilidad & Indicador \\
\hline $\begin{array}{l}\text { Conocer la tendencia } \\
\text { crítico-social de los } \\
\text { contenidos y la tendencia } \\
\text { pedagógica virtual } \\
\text { interpretar } \\
\text { el progreso histórico } \\
\text { de las } \\
\text { tecnologías, } \\
\text { utilizando sus } \\
\text { conceptos y } \\
\text { semántica }\end{array}$ & $\begin{array}{l}\text { teorías de la criticidad y } \\
\text { teorías de complejidad }\end{array}$ & $\begin{array}{l}\text { Elaborar una clase con ayuda de los } \\
\text { recursos de las tecnologías (ej: Word, } \\
\text { Power Point e Internet) exponiendo y } \\
\text { realizando análisis sobre estas teorías }\end{array}$ \\
\cline { 2 - 3 } & $\begin{array}{l}\text { Tener una visión histórica significa la } \\
\text { sociedad de la } \\
\text { información y del } \\
\text { conocimiento }\end{array}$ & $\begin{array}{l}\text { Realizar una búsqueda en la web sobre } \\
\text { los principales sitios que contienen el } \\
\text { tema sociedad de la información y del } \\
\text { conocimiento en forma de artículo o } \\
\text { entrevista }\end{array}$ \\
\hline
\end{tabular}




\begin{tabular}{|c|c|c|}
\hline & $\begin{array}{l}\text { Comprender el término } \\
\text { "tecnologías" }\end{array}$ & $\begin{array}{l}\text { Realizar un estudio sobre los términos } \\
\text { conocidos y plasmarlos en un } \\
\text { documento público }\end{array}$ \\
\hline & $\begin{array}{l}\text { Construir un glosario de } \\
\text { términos que forman parte } \\
\text { del vocabulario de uso de } \\
\text { las tecnologías }\end{array}$ & $\begin{array}{l}\text { Buscar en Internet lectura de textos de } \\
\text { autores que traten el tema virtual. } \\
\text { Analizar y comprender los conceptos, } \\
\text { sus elementos y características }\end{array}$ \\
\hline & $\begin{array}{l}\text { Utilizar los términos } \\
\text { estudiados sobre } \\
\text { tecnologías en el } \\
\text { vocabulario hablado y } \\
\text { escrito }\end{array}$ & $\begin{array}{l}\text { Analizar las ventajas y desventajas del } \\
\text { uso del computador en educación: por } \\
\text { qué es necesario y cuál es la diferencia } \\
\text { significativa para el proceso de } \\
\text { enseñanza y aprendizaje }\end{array}$ \\
\hline \multirow{3}{*}{$\begin{array}{l}\text { Tener como } \\
\text { referencia } \\
\text { de trabajo educativo } \\
\text { el } \\
\text { modelo teórico para } \\
\text { la } \\
\text { educación } \\
\text { fundamentado } \\
\text { epistemológicamente } \\
\text { en } \\
\text { la virtualidad }\end{array}$} & $\begin{array}{l}\text { Definir y analizar lo } \\
\text { virtual y la virtualidad en } \\
\text { sus bases teóricas }\end{array}$ & $\begin{array}{l}\text { Elaborar acciones de clase, basándose } \\
\text { en las características y elementos que } \\
\text { conocen el paradigma de la virtualidad }\end{array}$ \\
\hline & $\begin{array}{l}\text { Tener como concepto el } \\
\text { porqué del uso del } \\
\text { computador en la } \\
\text { educación, cuestionar y } \\
\text { analizar su función. Él es } \\
\text { la respuesta, pero ¿cuál es } \\
\text { la pregunta? }\end{array}$ & $\begin{array}{l}\text { Elaboración de materiales para la } \\
\text { acción en clase, utilizando aplicaciones } \\
\text { del computador (Word, Power Point, } \\
\text { etc) contemplando temas amplios y no } \\
\text { solamente los involucrados en } \\
\text { disciplinas, permitiendo que el alumno } \\
\text { pueda aprender a conectar las } \\
\text { informaciones y realizar, de esa } \\
\text { manera, un trabajo interdisciplinario }\end{array}$ \\
\hline & $\begin{array}{l}\text { Conocer los elementos y } \\
\text { las características } \\
\text { específicas del paradigma } \\
\text { de la virtualidad para la } \\
\text { educación }\end{array}$ & $\begin{array}{l}\text { Utilizar Internet para buscar } \\
\text { información, aprender los mecanismos } \\
\text { de búsqueda por los bancos de datos y } \\
\text { elaborar rutas de investigación }\end{array}$ \\
\hline \multirow{2}{*}{$\begin{array}{l}\text { Desarrollar acciones } \\
\text { pedagógicas para } \\
\text { la construcción del } \\
\text { conocimiento } \\
\text { utilizando } \\
\text { el computador y sus } \\
\text { recursos para el } \\
\text { proceso de } \\
\text { enseñanza y } \\
\text { aprendizaje }\end{array}$} & $\begin{array}{l}\text { Elaborar materiales } \\
\text { educativos para uso en el } \\
\text { proceso de la enseñanza y } \\
\text { aprendizaje que tengan } \\
\text { como referencia el } \\
\text { paradigma de la } \\
\text { virtualidad }\end{array}$ & $\begin{array}{l}\text { Elaboración de materiales para la } \\
\text { acción en clase, utilizando aplicaciones } \\
\text { del computador (Word, Power Point, } \\
\text { etc) contemplando temas amplios y no } \\
\text { solamente los involucrados en } \\
\text { disciplinas, permitiendo que el alumno } \\
\text { pueda aprender a conectar las } \\
\text { informaciones y realizar, de esa } \\
\text { manera, un trabajo interdisciplinario }\end{array}$ \\
\hline & $\begin{array}{l}\text { Conocer las aplicaciones } \\
\text { del computador para la } \\
\text { búsqueda de información } \\
\text { y ser capaz de realizar este } \\
\text { trabajo a través del uso de } \\
\text { la web }\end{array}$ & $\begin{array}{l}\text { Utilizar Internet para buscar } \\
\text { información, aprender los mecanismos } \\
\text { de búsqueda por los bancos de datos y } \\
\text { elaborar rutas de investigación }\end{array}$ \\
\hline
\end{tabular}




\begin{tabular}{|c|c|c|}
\hline \multirow{4}{*}{$\begin{array}{l}\text { El manejo, la } \\
\text { agilidad } \\
\text { y el conocimiento de } \\
\text { algunas de las } \\
\text { aplicaciones } \\
\text { (software) del } \\
\text { computador } \\
\text { y sus secuencias } \\
\text { básicas } \\
\text { de uso }\end{array}$} & $\begin{array}{l}\text { Comprender la definición } \\
\text { y el uso del entorno de } \\
\text { trabajo del sistema } \\
\text { operacional que será } \\
\text { utilizado en su estructura } \\
\text { de imágenes y las } \\
\text { funciones para abrir y } \\
\text { cerrar programas }\end{array}$ & $\begin{array}{l}\text { Elaboración de ejercicios para } \\
\text { aprendizaje, de forma simple, de esas } \\
\text { aplicaciones, sin mayores detalles, } \\
\text { centrados solamente en su función } \\
\text { central }\end{array}$ \\
\hline & $\begin{array}{l}\text { Perfeccionar las } \\
\text { posibilidades de uso de las } \\
\text { aplicaciones en funciones } \\
\text { más específicas }\end{array}$ & $\begin{array}{l}\text { Las actividades deben atender al } \\
\text { desarrollo de otras funciones de la } \\
\text { aplicación con más detalles y } \\
\text { perfeccionamiento del trabajo }\end{array}$ \\
\hline & $\begin{array}{l}\text { Ayudar a los estudiantes, } \\
\text { en el contexto de sus } \\
\text { asignaturas, a alcanzar } \\
\text { habilidades en el uso de } \\
\text { las TIC }\end{array}$ & $\begin{array}{l}\text { Seleccionar varias herramientas } \\
\text { específicas de las TIC para una } \\
\text { asignatura y pedir a los participantes } \\
\text { que identifiquen los estándares } \\
\text { específicos del plan de estudios } \\
\text { asociados a esas herramientas al igual } \\
\text { que discutan cómo éstos se pueden } \\
\text { apoyar en las TIC }\end{array}$ \\
\hline & $\begin{array}{l}\text { Utilizar las TIC para } \\
\text { evaluar la adquisición de } \\
\text { conocimientos, en } \\
\text { asignatura, por parte de } \\
\text { los estudiantes e } \\
\text { informarles sobre sus } \\
\text { progresos utilizando } \\
\text { evaluaciones tanto } \\
\text { formativas como } \\
\text { sumativas (acumulativa) }\end{array}$ & $\begin{array}{l}\text { Proponer a los participantes que } \\
\text { preparen un proyecto de clase sobre un } \\
\text { tema específico de una asignatura que } \\
\text { incluya alguna instrucción sobre la } \\
\text { utilización de las TIC. Más } \\
\text { concretamente: procesadores de texto, } \\
\text { navegadores de Internet, correo } \\
\text { electrónico, blogs, wikis y otras } \\
\text { tecnologías emergentes. Pedirles } \\
\text { además que se los presenten a sus } \\
\text { colegas y que les enseñen habilidades } \\
\text { en estas herramientas }\end{array}$ \\
\hline
\end{tabular}

Fuente: Vieira (2007).

\section{Impacto de las TIC en la educación superior}

Según Abdala (2004), bajo la denominación de evaluación de impacto se entiende el proceso evaluatorio orientado a medir los resultados de las intervenciones en cantidad, calidad y extensión, según las reglas preestablecidas. La medida de los resultados, característica principal de la evaluación de impacto, permite comparar el grado de realización alcanzado con el deseado. Se compara, de esta forma, la planeación con el resultado de la ejecución. La evaluación de impacto abarca todos los efectos secundarios a la planeación y a la ejecución: específicos y globales; buscados (según los objetivos) o no; positivos, negativos o neutros; directos o indirectos (la puesta 
en marcha del programa puede generar por sí misma efectos sobre quienes están directamente involucrados, hasta toda la sociedad).

Durante décadas, la idea predominante era "evaluar es medir", dándole peso sólo a las dimensiones e indicadores cuantitativos (Cabero \& López, 2009). Actualmente, la evaluación de impacto es valorada como un proceso amplio y global, en el que al abordaje cuantitativo se agregan técnicas cualitativas. 2004):

La evaluación de impacto, al medir los resultados, permite (Abdala,

- Registrar y analizar todas las experiencias (positivas y negativas), mediante la comparación en el grupo control, sistematizándola.

- Evaluar el contexto socioeconómico y político en que se da la experiencia.

- Identificar los actores involucrados y su peso específico en los resultados.

- $\quad$ Estudiar la articulación interinstitucional y público-privada.

- Ofrecer estudios de costo-beneficio.

- Concertar aportes de los técnicos en gestión, mediante la difusión de la información proveniente de la evaluación y su posterior discusión entre todos los responsables de la gestión.

- Informar de forma clara y objetiva a los responsables de la toma de decisiones sobre la marcha de los programas; esta retroalimentación promueve el reforzamiento institucional.

\section{Las TIC frente a los retos de la educación superior}

Toda esta revolución tecnológica ha generado nuevas y mayores oportunidades de interacción que requieren, a su vez, una capacidad de adaptación, tanto de los individuos como de las instituciones (Salinas, 2004). Frente a este panorama, las instituciones de educación superior han venido experimentando cambios y realizando iniciativas para convertirse en contextos altamente tecnológicos.

Este tipo de instituciones han entendido que el uso pertinente de las TIC y los procesos de innovación que las incorporan les permiten alcanzar sus principales objetivos formativos: promover que los estudiantes desarrollen conocimientos científicos rigurosos, integrados y aplicados en su desempeño profesional, fomentar en los alumnos la capacidad de ser aprendices autónomos y autorregulados y participar en la consolidación de ciudadanos críticos, reflexivos, creativos y responsables (Monereo \& Pozo, 2003; Pozo, 2008; UNESCO, 1998).

Entonces, estos objetivos propios de la educación superior y los cambios institucionales realizados que incorporan a las TIC para lograr dichos 
fines se alinean con dos perspectivas contemporáneas sobre el aprendizaje. Por un lado, se relacionan con el constructivismo sociocultural (Coll, 2002; Coll, Mauri \& Onrubia, 2008), enfoque que enfatiza el carácter social del aprendizaje, pues propone que el alumno construye conocimientos de forma compartida con sus pares y el docente, y con el apoyo de las herramientas culturales de su entorno. En este caso, los dispositivos y aplicaciones digitales actuarían como instrumentos mediadores del aprendizaje y del funcionamiento cognitivo (Díaz-Barriga, 2005).

Por otro lado, se asocia con el conexionismo (Siemens, 2004), que considera a la tecnología de esta era digital como un factor clave en la creación de vínculos entre diversas fuentes de información, así como el desarrollo de dichas redes para facilitar el aprendizaje continuo. En esta dinámica, la función del docente consiste en generar oportunidades de aprendizaje, en el que el alumno pueda cuestionarse qué es lo que ya conoce acerca del tema a desarrollar, buscar nueva información, y trabajar en colaboración con otros para resolver problemas y tomar decisiones. De este modo, el docente es responsable de guiar a sus alumnos facilitándoles el acceso a recursos que les permitan explorar y elaborar nuevos conocimientos, lo cual fomenta el desarrollo de habilidades y destrezas. Luego, como fin último, los estudiantes serán capaces de utilizar dichas herramientas de manera autónoma, estratégica y eficiente (Díaz-Barriga, 2005).

De esta manera, surgen las oportunidades necesarias para el desarrollo de las competencias requeridas en la era digital, entre las que se contempla el aprendizaje multifacético, informal y permanente. En esto último, cobra relevancia el llamado aprendizaje invisible (Cobo \& Moravec, 2011), pues la creciente accesibilidad de las tecnologías favorece la integración de la educación formal con la no formal, lo que implica procesos de generación, actualización, y aplicación de conocimientos significativos y contextualizados.

De este modo, el aprendizaje cotidiano, a veces imperceptible, que aprovecha el potencial de las TIC, facilita el desarrollo de diversas capacidades de experimentación, innovación y colaboración. Justamente en este entorno de cambios propios del siglo XXI, hoy no sólo miles de personas pueden acceder a una mayor cantidad de información, sino también interactuar activamente con su medio, creando, adaptando y compartiendo el conocimiento en tiempo real (Escofet et al., 2011; Pedró, 2011; Solomon \& Schrum, 2007). Por tales razones, se afirma que las nuevas tecnologías son participativas por naturaleza, pues, actualmente, los usuarios "no solo leen, también discuten, comentan, valoran, opinan, proponen, anuncian, enlazan, escriben, publican, intercambian, escogen, corrigen, comparten; es decir, participan activamente" (Nafría, 2007). 


\section{Resultados}

Gráfico $N^{\circ}$ 1. Edad del docente

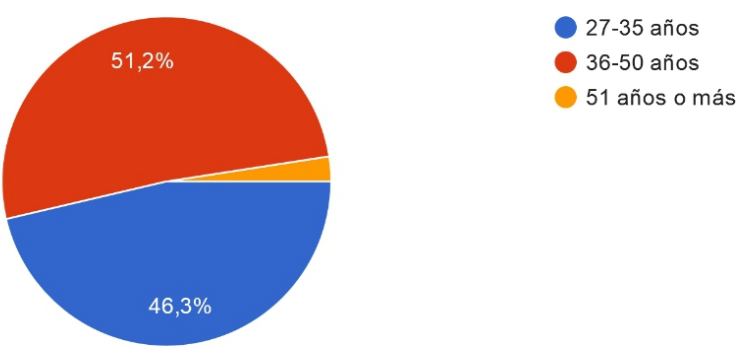

Elaborado por: Autores

Los resultados de la presente gráfica evidencian que de 117 docentes que trabajan en la Unidad de Admisión y Nivelación de la Escuela Superior Politécnica de Chimborazo existe un 51,2\% de personas cuya edad oscila entre 36 y 50 años. También se demuestra que existen personas jóvenes con una edad entre 27 y 35 años representando un 46,3\%, que apenas el 2,5\% personas superan los 51 años de edad, y finalmente que más de un $90 \%$ de las personas tienen una edad joven, la cual permite que manejen de manera adecuada las TICs.

Gráfico $\mathrm{N}^{\circ}$ 2. Herramientas tecnológicas de uso frecuente

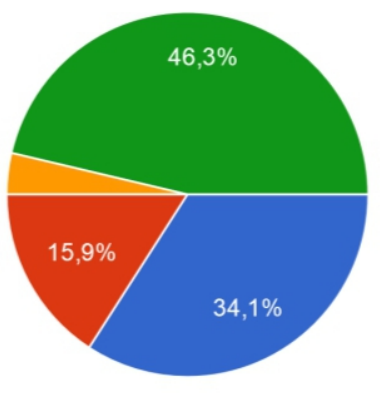

Proyector - Computador - uso de software

Acceso a internet

Redes sociales (Facebook,

Whats App, otros)

Aula virtual con contenidos multimedia

Elaborado por: Autores

Los resultados de la presente gráfica demuestran que las herramientas tecnológicas de uso frecuente por parte de los docentes de Unidad de Admisión y Nivelación, se demuestra que el 46,3 \% utiliza el aula virtual con contenidos multimedia institucionales como herramienta complementaria en la formación de sus estudiantes, el 34,1 \% continúa con el uso del Proyector, computador y software como apoyo en sus actividades académicas, el 15,9\% utiliza el internet como herramienta TIC, y apenas el 3,7\% utiliza las redes 
sociales como herramienta tecnológica en sus procesos de enseñanza aprendizaje.

Gráfico $N^{\circ}$ 3. Calidad de Acceso a las TIC en la ESPOCH

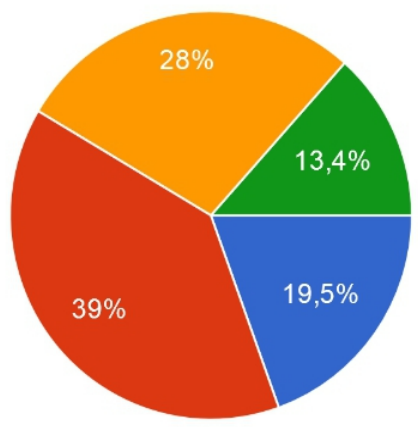

Excelente

Muy buena

Buena

Regular

\section{Elaborado por: Autores}

Los resultados de la presente gráfica indican que según el criterio de los docentes el $39 \%$ manifiesta que la calidad de acceso a las TIC en la ESPOCH en Muy Buena, el $28 \%$ indica que es Buena, el 19,5\% dice que es excelente el acceso, y un 13,4\% que equivale a 11 docentes indica que el acceso a las TIC es regular.

Gráfico $N^{\circ}$ 4. Acceso a Internet en la ESPOCH
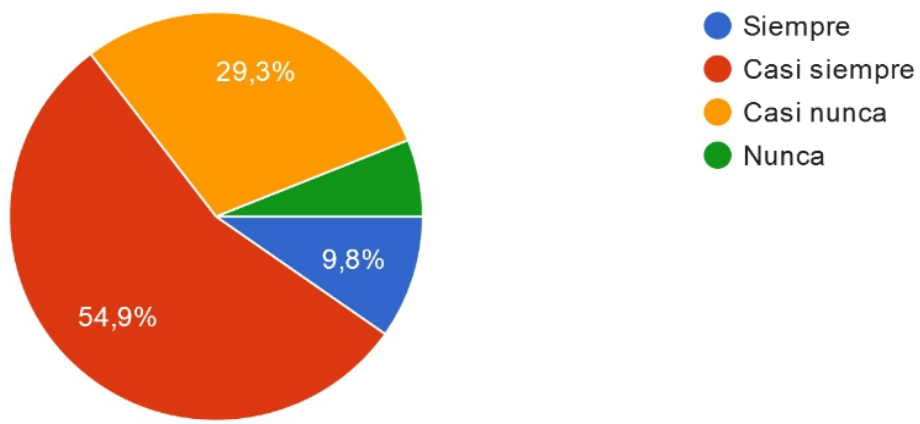

\section{Elaborado por: Autores}

Los resultados de la presente gráfica demuestra que los docentes de la Unidad de Admisión y Nivelación indican que casi siempre en un 54,9 \% tienen acceso a Internet en el campus institucional sin ningún inconveniente, el 29,3\% ,representado por 25 docentes, manifiesta que casi nunca cuentan con servicio de internet, apenas un $9,8 \%$ indica que siempre cuentan con servicio de Internet, pero $6 \%$ indican que nunca cuentan con el servicio de Internet, lo cual dificultaría el desarrollo de sus actividades en el proceso de enseñanza aprendizaje en la Unidad de Admisión y Nivelación de la ESPOCH. 


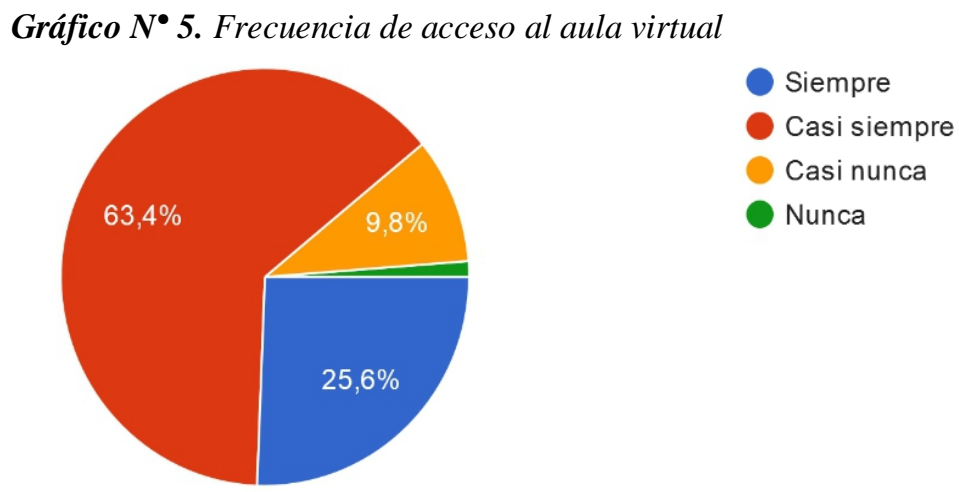

Elaborado por: Autores

Los resultados de la presente gráfica permiten determinar que los docentes utilizan casi siempre el aula virtual como una herramienta complementaria de las TIC en el proceso de enseñanza aprendizaje en un 63,4 $\%, 25,6 \%$ siempre realizan un trabajo de acampamiento con los estudiantes a través del aula virtual, el 9,8\% de los docentes casi nunca utilizan el aula virtual, únicamente el 1,2\% de los docentes nunca ha utilizado el aula virtual, lo que evidencia que al menos $89,8 \%$ de la totalidad de docentes han utilizado al menos una vez el aula virtual.

Gráfico $N^{\circ}$ 6. Criterios para elección de una herramienta tecnológica

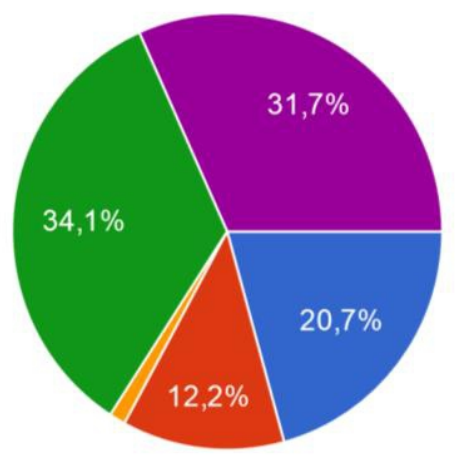

- Los contenidos de la asignatura que imparte a los estudiantes

- Atender a las necesidades académicas, profesionales y socia...

- Los conocimientos que usted tenga sobre la herramienta

- Que los estudiantes realmente aprendan y se motiven

- Que se logre una comunicación más efectiva y más claridad de co...

\section{Elaborado por: Autores}

Los resultados de la presente gráfica determinan que los docentes, al momento de elegir una herramienta tecnológica para su aula, el 34,1\% se basa en que los estudiantes realmente aprendan y se motiven, el $31,7 \%$ se preocupa de que se logre una comunicación más efectiva y que el contenido sea claro, el $20,7 \%$ prefieren los contenidos de la asignatura que se imparten a los estudiantes, un $12,2 \%$ busca atender a las necesidades académicas y profesionales y sociales de los estudiantes y finalmente el 1,3\% se preocupa de los conocimientos que él como docente tiene de la herramienta tecnológica. 
Gráfico $N^{\circ}$ 7. Seguimiento del acceso a los recursos tecnológicos

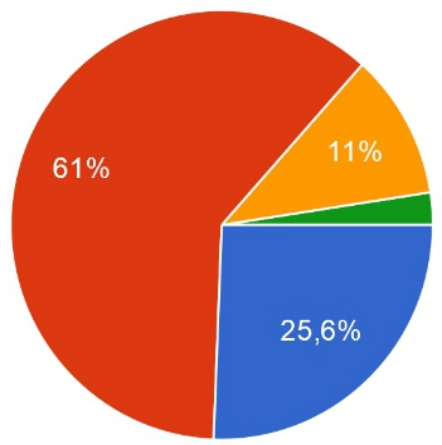

Siempre

Casi siempre

Casi nunca

Nunca

Elaborado por: Autores

Los resultados de la presente gráfica demuestran que el $61 \%$ de los docentes realiza un seguimiento de acceso a los recursos que suben en su aula para verificar si es descargado y utilizado por sus estudiantes, el 25,6\% siempre revisa su aula virtual para monitorear el acceso y el $11 \%$ no revisa si los estudiantes acceden a los contenidos del aula virtual.

Gráfico $N^{\circ} 8$. Ventajas de trabajo con las TIC en el aula (presencial o virtual)

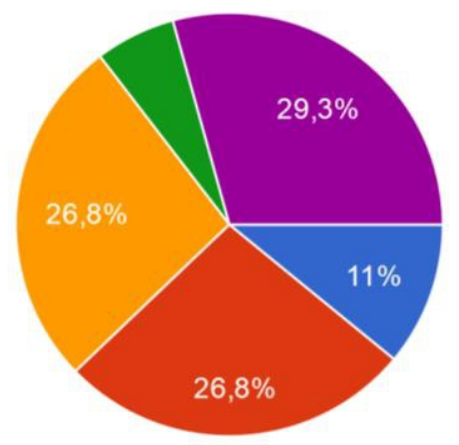

Es algo innovador

El trabajo se facilita mucho con su uso (ahorro de tiempo)

Se puede ayudar al estudiante en cualquier momento

El uso de bibliotecas virtuales

- Las destrezas que pueden desarrollarse a través de ellas

\section{Elaborado por: Autores}

Los resultados de la presente gráfica determinan que el 29,3\% de los docentes indica que una de las ventajas de trabajar con las TIC en el aula (presencial o virtual) es las destrezas que se pueden desarrollar a través de ellas por parte de los estudiantes, un $26,8 \%$ indica que el tiempo de trabajo se puede disminuir, el $26,8 \%$ indica que se puede tutorear a los estudiantes en cualquier instante de tiempo sin necesidad de la presencia física, y el $11 \%$ indica que su uso es algo innovador. 
Gráfico $\boldsymbol{N}^{\circ}$ 9. Mejoramiento en el proceso de aprendizaje a través de las TIC

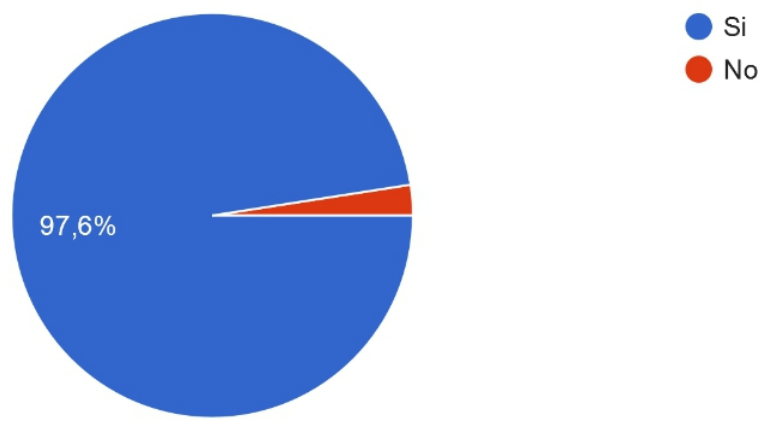

Elaborado por: Autores

Los resultados de la presente gráfica determinan que el 97,6 \% de los docentes indica que el uso de las TIC puede mejorar el proceso de enseñanza aprendizaje, en tanto que un $2,4 \%$ dice lo contrario.

Gráfico $N^{\circ}$ 10. Participación en procesos de capacitación en temas de enseñanzaaprendizaje virtual (e-learning)

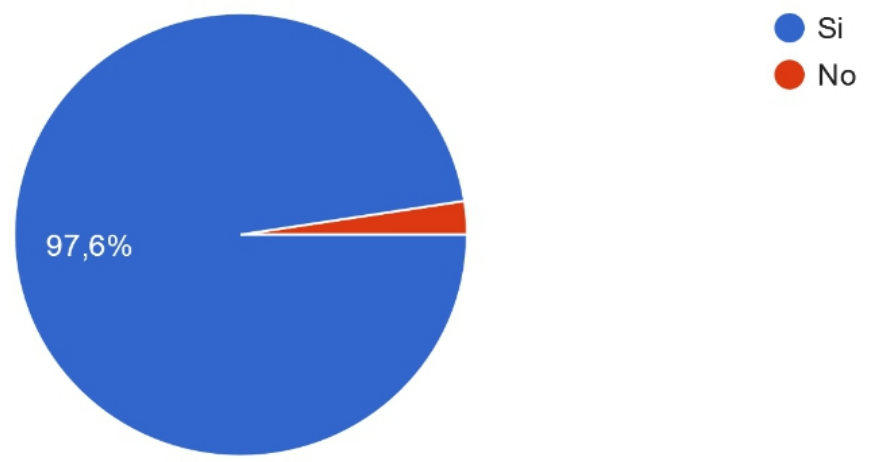

Elaborado por: Autores

Los resultados de la presente gráfica muestran que el 97,6\% de los docentes está dispuesto a participar en procesos de capacitación en temas de enseñanza aprendizaje virtual (e-learning) para mejorar, en tanto que un 2,4 $\%$ dice lo contrario.

\section{Discusión}

El presente trabajo de investigación se desarrolló en la Escuela Superior Politécnica de Chimborazo, campus matriz de la ciudad de Riobamba en la Unidad de Admisión y Nivelación en el período académico 2018 2-S correspondiente a los meses de Octubre 2018 - Febrero 2019.

La Unidad Académica en mención en ese período contó con 77 docentes contratados, para lo cual se determinó el tamaño de la muestra con un margen de error maximo admitido del 5\%. Se encuestó a 69 estudiantes de 
la Unidad de Admisión y Nivelación registrando un nivel de confianza del 99\%, para lo cual se utilizó una encuesta con 10 preguntas de opción simple realizada a través de las redes sociales.

Los objetivos que se perseguían fueron los siguientes:

$\checkmark \quad$ Conocer la edad promedio de los docentes de la Unidad de Admisión y Nivelación de la Escuela Superior Politécnica de Chimborazo, para determinar cual es el contenido multimedia de su prefencia de acuerdo a cada segmento.

$\checkmark \quad$ Conocer las herramientas de uso frecuente de los docentes para el desarrollo de sus clases.

$\checkmark \quad$ Determinar la calidad de acceso a las TIC en la Escuela Superior Politécnica de Chimborazo en sus campus matriz.

$\checkmark \quad$ Conocer si existe el acceso a Internet en el campus y si este es el adecuado y brinda las facilidades necesarias para el uso de las TIC en la Educación Superior.

$\checkmark \quad$ Determinar la frecuencia de uso de la plataforma virtual institucional, la ccual es el la herramienta tecnológica de preferencia de los docentes, el contenido que los docentes suben a su aula y el seguimiento que realizan alos estudiantes de acuerdo al uso del aula por parte de los mismos.

El tipo de muestreo utilizado aleatorio simple a través del calculo del tamaño óptimo de la muestra (para la estimación de proporciones, bajo el supuesto de que $\mathrm{p}=\mathrm{q}=50 \%$ ), en donde ( $\mathrm{p}$ ) es la probabilidad de éxito o proporción esperada, (q) probabilidad de fracaso, (N) el tamaño de la población y (z) el nivel de confianza con lo que determinamos una muestra representativa de cuántos docentes tendríamos que evaluar para conocer el uso que le brindan a la plataforma virtual con fines académicos.

La Escuela Superior Politécnica de Chimborazo utiliza como plataforma virtual la herramienta Moodle en su versión 3.2, la cual se encuentra alojada en el Departamento de Tecnologías de la Información y Comunicación en donde se alojan las aulas virtuales de las 7 facultades con 29 carreras, las 2 extensiones con 4 carreras cada una y las aulas de la Unidad de Admisión y Nivelación.- Dicha plataforma ha sido adaptada a la necesidad institucional, por lo que la misma ya no es compatible con las actualizaciones oficiales. 
Gráfico $N^{\circ}$ 11. Plataforma virtual de la ESPOCH

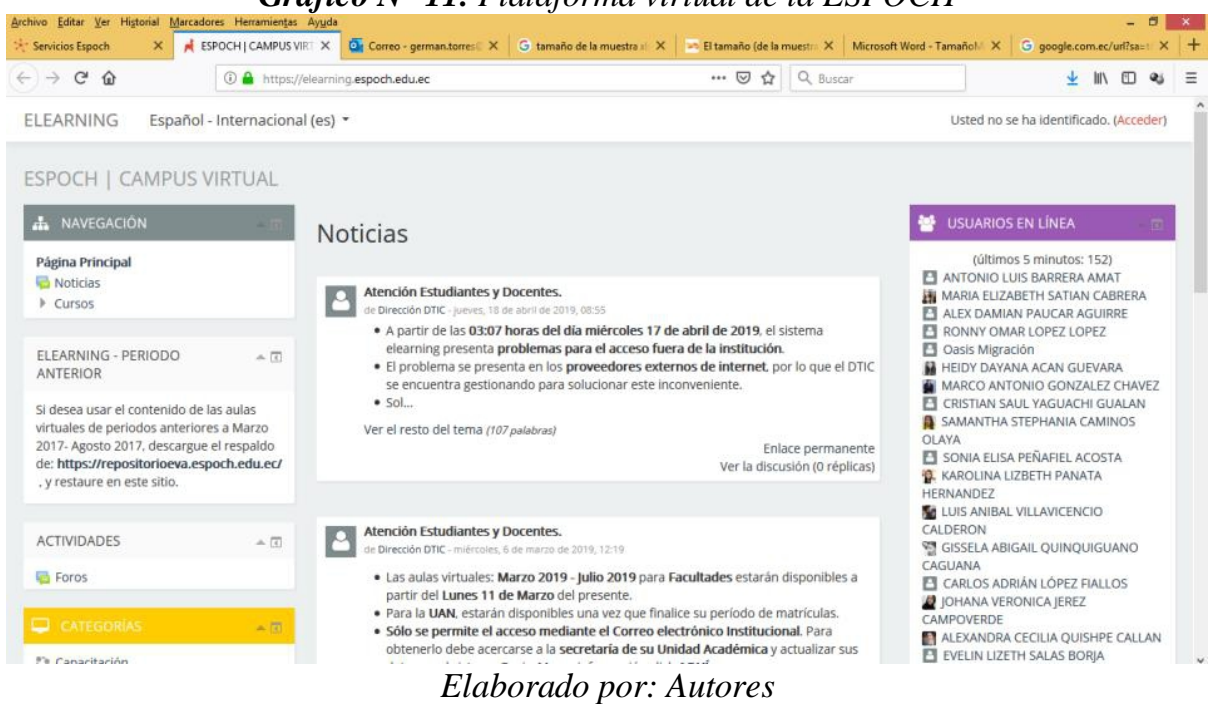

La Unidad de Admisión y Nivelación maneja un total de 345 aulas virtuales en un total de 69 paralelos con un total de 5 aulas por paralelo, las cuales se encuentran organizadas por profesor, paralelo y asignatura. Estas permiten tener tiempos de respuesta inmediatos con los estudiantes y son una herramienta de apoyo al proceso de enseñanaza aprendizaje,. El 91\% de los docentes utiliza la plataforma virtual institucional.

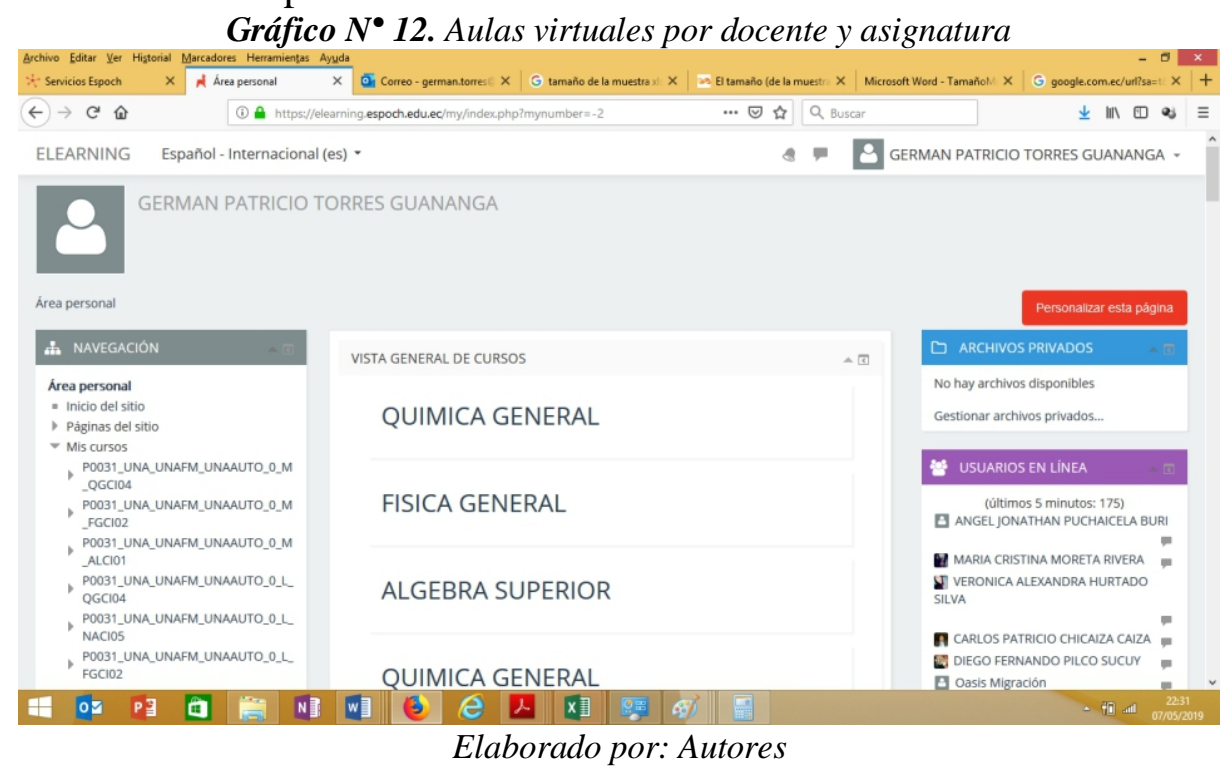

\section{Conclusiones}

Pese a que la edad del 51,2\% de los docentes de la Unidad de Admisión y Nivelación de la ESPOCH oscila entre 36-50 años, únicamente el 46,3\% 
prefiere utilizar el aula virtual con contenidos multimedia, pero existe todavía un $34,1 \%$ de docentes que prefieren seguir utilizando su proyecto y el computador como herramienta de apoyo al desarrollo de sus actividades académicas. Por tal motivo,se hace necesario capacitar a los docentes para mejorar en lo que respecta al uso de las TIC en la Educación Superior.

El acceso a las TIC en la ESPOCH es muy bueno en un 39\%, debido que únicamente el $54,9 \%$ de los docentes de la unidad casi siempre tiene acceso a Internet, un 30\% indica que casi nunca tiene acceso a Internet por lo que no tienen tiempos de respuesta adecuados a las inquietudes de los estudiantes sobre los temas de las asignaturas a través del uso de la plataforma virtual, perdiendo así su nivel de confianza.

Los docentes acceden a su aula virtual casi siempre en un 63,4\%, lo que permite conocer las inquietudes que pueden ser planteadas por los estudiantes. Además, el 34,1\% se preocupa de que los estudiantes, a través de su aula virtual, aprendan y se motiven, y el $61 \%$ realiza un seguimiento del acceso a los recursos que suben a su aula virtual y si este no es descargado y utilizado por sus estudiantes, lo que permite que los estudiantes siempre revisen el contenido de la asignatura a través de su aula.

El 97,6 \% de los docentes están convencidos de que el uso de las TIC puede mejorar el proceso de enseñanza aprendizaje en la Unidad de Admisión y Nivelación de la Escuela Superior Politécnica de Chimborazo en su campus matriz, por lo que el mismo número de docentes está dispuesto a capacitarse en temas de TIC en la Educación Superior.

\section{References:}

1. Abdala, E. (2004): Manual para la evaluación de impacto en programas de formación para jóvenes.Montevideo.

2. Area, M. (2000): Problemas y retos educativos ante las tecnologías digitales en la sociedad de la información, Quaderns Digitals, 28. Disponible en: http://www.quadernsdigitals.net/index.php? accionMenu=hemeroteca. VisualizaArticuloIU.visualiza\&articulo_id $=284$

3. Area, M. (2007). Algunos principios para el desarrollo de buenas prácticas pedagógicas con las TICs en el aula. Comunicación Y Pedagogía: Nuevas Tecnologías Y Recursos Didácticos, 222, 42-47. Disponible en: http://manarea.webs.ull.es/wpcontent/uploads/2010/06/CyPbuenaspracticasTIC.pdf

4. Banannan-Ritland, B. (2008). Handbook of Design Research Methods in Education: Innovations in Science, Technology, Enginnering and Mathematics Learning and Teaching. New York: Routledge.

5. Bazzo, M. (2010). Integração de Tecnologias de Informação e Comunicação no Ensino: Contribuições dos Modelos de Difusão e 
Adoção de Inovações para o campo da Tecnologia Educacional. Revista Latinoamericana de Tecnología Educativa RELATEC, 9 (1), 89106. [en línea] Disponible en: http:// campusvirtual.unex.es/cala/editio/).

6. Belloch, C. (2012). Las Tecnologías de la Información y Comunicación en el aprendizaje. Disponible en: http://www.uv.es/bellochc/pedagogia/EVA1.pdf

7. Benvenuto, A. (2003). Las tecnologías de información y comunicaciones (TIC) en la docencia universitaria.Theoria, 12(1), 109-118.

Disponible

en: http://www.redalyc.org/articulo.oa?id=29901210.

8. Cabero, J. (2004). Reflexiones sobre las tecnologías como instrumentos culturales.

9. Cabero, J. \& López, E. (2009). Construcción de un instrumento para la evaluación de las estrategias de enseñanza de cursos telemáticos de formación universitaria. Edutec: Revista Electrónica de Tecnología Educativa, $\quad \mathrm{N}^{\circ} 28$ [en línea]. Disponible en: http://edutec.rediris.es/revelec2/revelec28002F

10. Cascales, A. (2017). Metodología y Tecnologías de la Información y Comunicación: Webquest.

11. Castells, M. (2001). La era de la información. Vol 1. La Sociedad Red. Segunda edición, p 60, Madrid, España, Alianza Editorial S.A.

12. CEPAL (2003). "Los caminos hacia una sociedad de la información en América Latina y el Caribe", Santiago de Chile, julio de 2003.

13. Cobo, C. \& Moravec, J. (2011). Aprendizaje invisible. Hacia una nueva ecología de la educación. Bercelona: Colección Transmedia XXI. Disponible en: http://www.aprendizajeinvisible.com/download/AprendizajeInvisible. pdf.

14. Coll, C. (2002). El constructivismo en el aula. Barcelona: Graó. 2004 Psicología de la educación y prácticas educativas mediadas por las tecnologías de la información y la comunicación: una mirada constructivista. Revista Electrónica Sinéctica, 25, 1-24. Disponible en: http://www.virtualeduca.org/ifd/pdf/cesar-coll-separata.pdf

15. Coll, C., Mauri, M., \& Onrubia, J. (2008). Análisis de los usos reales de las TIC en contextos educativos formales: una aproximación sociocultural. Revista Electrónica de Investigación Educativa, 10(1), 1-18. Disponible en: http://redie.uabc. mx/index.php/redie/article/view/177/307.

16. De Pablos Pons, J. \& Jiménez Cortés, R. (2007). Buenas prácticas con TIC apoyadas en las Políticas Educativas: claves conceptuales y derivaciones para la formación en competencias ECTS, Revista 
Latinoamericana de Tecnología Educativa, 6 (2), 15-28. [http://campusvirtual.unex.es/cala/editio/]

17. Díaz-Barriga, F. (2005). Principios de diseño instruccional de entornos de aprendizaje apoyados con TIC: un marco de referencia sociocultural y situado. Revista Tecnología y Comunicación Educativas, 41, 4-16. Disponible en: http://investigacion.ilce.edu.mx/stx.asp?id=2333\&db=\&ver

18. Domínguez, R. (junio, 2011). Formación competencia y actitudes sobre las TIC del profesorado de secundaria: un instrumento de evaluación.

http://www.ugr.es/ sevimeco/revistaeticanet/numero10/Articulos/For mato/articulo6.pdf

19. Escofet, A., García, I. \& Gros, B. (2011). Las nuevas culturas de aprendizaje y su incidencia en la educación superior. Revista mexicana de investigación educativa, 16(51), 1177-1195. Disponible en: http://www.redalyc.org/articulo. oa?id=14019203008

20. Gómez, J. \& Cano, J. (2011). El pensamiento docente y su influencia en la implantación de las tecnologías de la información y la comunicación en el aula: desafíos y oportunidades. Contextos, Educativos, $14 . \quad$ Disponible en: http://publicaciones.unirioja.es/ojs2.4.2/index.php/contextos/article/vi ew/640

21. Gonzales Nelo, Trelles César, \& Mora Janeth (2017). Manejo Docente de las Tecnologías de la Información y Comunicación. Cuenca, Ecuador.

22. Hilbert, M. \& Katz, J. (2003). "Building an Information Society: A Latin American and Caribbean Perspective", Santiago de Chile, CEPAL-ECLAC.

23. Jaramillo, P., Castañeda, P. \& Pimienta, M. (2009). Qué hacer con la tecnología en el aula: inventario de usos de las TIC para aprender y enseñar. Educación y Educadores, 12(2), 159-179. Disponible en: http://www.redalyc.org/pdf/834/83412219011. pdf

24. Kumari, S. \& D'Souza, F. (2016). Secondary school teachers' digital literacy and use of ICT in teaching and leraning, I(I), 141-146. Disponible en: http://ijcrd.dvpublication.com/2016/12/23/secondaryschool-teachers-digital-literacy-anduse-of-ict-in-teaching-andlearning/

25. Linares, J. (2004). "Principios de una ética para el mundo tecnológico", México, UNAM.

26. Lira, A. (2005). La tecnología de la información y comunicación: estudio económico. 
27. López de la, M.C. \& Chávez, J.E. (julio-diciembre, 2013). La formación de profesores universitarios en la aplicación de las TIC. Sintética.

28. Márquez, A. \& Ramón, P. (junio, 2013). Uso de las Tecnologías de la Información y Comunicación en los docentes de la UJAT. Red Durango de Investigadores Educativos (ReDIE, A.C.). Recuperado de: http://redie.mx/librosyrevistas/libros/forma_doc.pdf

29. Martínez, F. \& Frendes, M. (Comp.). Nuevas tecnologías y educación, (pp. 15-19). Madrid: Pearson.

30. Ministerio de Educación (2007). Proyecto emergente de Unidades Educativas del Milenio y Establecimientos Réplica. Disponible en: http://educacion.gob.ec/proyecto-emergente-deunidades-educativasdel-milenio-y-establecimientos-replica/

31. Ministerio de Educación (2014). Docentes de Pastaza recibieron computadoras, p. 1. Disponible en: http://educacion.gob.ec/docentesde-pastaza-recibieron-computadoras/

32. Ministerio de Educación (2016). Currículo de los niveles de Educación Obligatoria. Disponible en: http://educacion.gob.ec/wpcontent/uploads/downloads/2016/08/Curriculov2.pdf

33. Monereo, C. \& Pozo, J.I. (2003). La universidad ante la nueva cultura educativa: enseñar y aprender para la autonomía. Madrid: Síntesis. 2007 Competencias para (con)vivir con el siglo XXI. Cuadernos de Pedagogía, 370, 12-18. Disponible en: http://www.documentacion.edex.es/docs/0401POZcom.pdf

34. Nafría, I. (2007). Web 2.0: el usuario, el nuevo rey de internet,Barcelona: Gestión 2000. North Central Regional Educational Laboratory (NCREL) \&Metiri Group. (2003). 21st century skills: literacy in thedigital age. Disponible en: http://pict.sdsu.edu/engauge21st.pdf

35. Pedraza, N., Farías G., Lavín, J. \& Torres, A. (2013). Las competencias docentes en TIC en las áreas de negocios y contaduría. Un estudio exploratorio en la educación superior. Perfiles educativos 35(139). Pp. 8-24. Disponible en: http://www.redalyc.org/articulo.oa?id=13225611011

36. Pedró, F. (2011). Tecnología y escuela: lo que funciona y por qué. Madrid: Fundación Santillana. Disponible en: http://www.stecyl.es/informes/Edu-Sociedad-Digital/TecnEscuela_documento_basico.pdf

37. Peñaherrera, M. (2012). Uso de Tic en Escuelas Públicas de Ecuador: Análisis, Reflexiones Y Valoraciones. EDUTEC. Revista Electrónica de Tecnología Educativa, 40, 1-16. Disponible en: http://www.edutec.es/revista/index.php/edutec-e/article/view/364 
38. Pozo, J.I. (2008). Aprendices y maestros: la psicología cognitiva del aprendizaje. Madrid: Alianza Editorial.

39. Salinas, J. (2004). Innovación docente y uso de las TIC en la enseñanza universitaria. Revista Universidad y Sociedad del Conocimiento, 1(1), 1-16. Disponible en: http://www.uoc.edu/rusc/dt/esp/salinas1104.pdf

40. Salinas, J. (2008). Innovación educativa y uso de las TIC. (Universidad Internacional de Andalucía, Ed.). Sevilla. Disponible en: http://dialnet.unirioja.es/servlet/articulo?codigo $=2906571$

41. Scheuermann, F., Kikis, K. \& Villalba, E. (2009). A framework for understanding and evaluating the impact of information and communication technologies in education. Centre for Research on Lifelong Learning (CRELL).

42. Serrano, A. \& Evelio, M. (2003). La brecha digital: mitos y realidades. Universidad Autónoma de Baja California. Departamento Editorial Universitaria. México.

43. Siemens, G. (2004). Connectivism: a learningtheoryforthe digital age. Disponible en: http://www.elearnspace.org/ Articles/connectivism.htm

44. Solomon, G. \& Schrum, L. (2007). Web 2.0: new tools, new schools. Washington D.C.: ISTE.

45. Tilve, M.D., Gewerc, A. \& Álvarez, Q. (2009). Proyectos de innovación curricular mediados por TIC: Un estudio de caso. Revista Latinoamericana de Tecnología Educativa RELATEC, 8 (1), 6581. [en línea] Disponible en: http:// campusvirtual.unex.es/cala/editio/ (consulta 2010, 29 de julio).

46. UNESCO (1998). Conferencia mundial sobre la educación superior. La educación superior en el siglo XXI: visión y acción. París: UNESCO. Disponible en: http://unesdoc.unesco.org/images/0011/001163/116345s.pdf

47. UNESCO (2013). Enfoques estratégicos sobre las TICS en educación e América Latina y el Caribe. Disponible en: http://www.unesco.org/new/fileadmin/MULTIMEDIA/FIELD/Santia go/ images/ticsesp.pdf

48. Wengslinky, H. (1998). Does It Compute? The Relationship Between Educational Technology and Student Achievement in Mathematics. Princeton. Disponible en: http://files.eric.ed.gov/fulltext/ED425191.pdf. 